\title{
The Rasch Model, Objective Measurement, Equating, and Robustness
}

\author{
Jeffrey A. Slinde \\ Science Research Associates, Inc. \\ Robert L. Linn \\ University of Illinois, Urbana-Champaign
}

This study investigated the adequacy of the Rasch model in providing objective measurement when equating existing standardized reading achievement tests with groups of examinees not widely separated in ability. To provide the context for the assessment of objectivity with the Rasch model, information relevant to several assumptions of the model was provided. An anchor test procedure was used to equate the various pairs of existing achievement tests. Despite the considerable lack of fit of the data to the model found for all tests used, the Rasch difficulty estimates were reasonably invariant for replications with random samples as well as samples that differed in ability by one grade level. Furthermore, with the exception of the data for one test pair and one grade level, the Rasch model using the anchor test procedure provided a reasonably satisfactory means of equating three test pairs on the log ability scale for the examinees at two grade levels.

Many authors (e.g., see the special Journal of Educational Measurement summer, 1977, issue on applications of latent trait models) have indicated that latent trait models offer a potentially good means of solving many difficult psychometric problems, such as vertical equating, tailored or adaptive testing, and criterion-referenced testing. The reason for this optimism is

APPLIED PSYCHOLOGICAL MEASUREMENT Vol. 3, No. 4 Fall 1979 pp. 437-452

(C) Copyright 1979 West Publishing Co. that objective measurement, which consists of person-free test calibration and item-free person measurement, is a consequence of such models. Person-free test calibration refers to the estimation of item parameters that are invariant for all groups of examinees. Item-free person measurement implies that except for errors of measurement, the same ability estimates would be obtained for an individual no matter which subset of calibrated items was used in the measurement. It is easy to see that if these two properties can reasonably be achieved, then the difficulties associated with many testing problems would disappear.

One of the simplest and most tractable latent trait models is the one-parameter logistic latent trait, or Rasch, model (Rasch, 1960). Only one item parameter-difficulty or location with respect to the item characteristic curve (ICC)-is specified by the Rasch model. The Rasch model does not include parameters for the lower asymptote or slope (discrimination) of the ICC. Like most of the latent trait models, the Rasch model also assumes that the items can be accounted for by only one latent trait or psychological function. In addition, the Rasch model, as well as other latent trait models, requires the tests to be unspeeded; otherwise, the probability of correctly answering the last items depends not only on the probability of success due to at- 
tempting the item but also on the probability of attempting the item.

Several studies (Anderson, Kearney, \& Everett, 1968; Rentz \& Bashaw, 1975; Tinsley \& Dawis, 1975) have investigated the person-free test calibration property of the Rasch model, reaching the conclusion that the difficulty estimates were relatively independent of the ability level of the calibration sample. The results of other studies (Tinsley \& Dawis, 1977; Whitely \& Dawis, 1974; Wright, 1968), which investigated the item-free person measurement property of the Rasch model, indicated that the Rasch ability estimates were reasonably independent of the difficulty of the items.

The extent of fit of data to the Rasch model with existing tests has received only limited attention in connection with the results concerning objective measurement, however. Also, crossvalidations of Rasch results were not reported in the studies dealing with item-free person measurement. That is, in all three studies the sample used to estimate the parameters was the same as that used to test the item-free person measurement claim of the Rasch model. In addition, the results of the Slinde and Linn studies (1978, 1979) suggested that item-free person measurement may not be realized with sets of multiplechoice items that differ markedly in difficulty.

In Slinde and Linn's studies, objective measurement was investigated within the context of the vertical equating problem by using crossvalidations of Rasch results for groups of quite different ability and for tests of quite different difficulty. Since extreme groups and tests were used, the test of the adequacy of the Rasch model for the vertical equating problem was acknowledged to be a severe one. Slinde and Linn also indicated that better results might be expected under such circumstances if an anchor test were used. Thus, it seems important and necessary to investigate further the claim for objective measurement with the Rasch model.

The primary purpose of this study, therefore, was to investigate the adequacy of the Rasch model in providing objective measurement when equating existing standardized reading achievement tests with groups of examinees not widely different in ability. More specifically, the assessment of objectivity with the Rasch model involved two considerations: (1) whether approximately equal item difficulty parameter estimates are obtained by the Rasch model when different (random and nonrandom) groups of examinees are used for the item calibration and (2) whether, on the average, a group of examinees (different from those used for the estimation) obtained the same ability estimates from two tests that differ in difficulty level. The first consideration refers to investigating the person-free item calibration property of the Rasch model, whereas the second consideration involves investigating the item-free person measurement property. To provide the context for the assessment of objectivity with the Rasch model, information relevant to several assumptions of the model was provided. Also, an anchor test procedure was used to equate the various pairs of existing achievement tests.

\section{Procedure}

To investigate whether objective measurement is achieved with the Rasch model, item response data were taken from the Anchor Test Study (ATS; Bianchini \& Loret, 1974). For the ATS a stratified random sample of students from the population of all fourth-, fifth-, and sixth-grade students in the United States was administered the reading comprehension and vocabulary subtests of two standardized achievement test batteries. A separate group of examinees was administered each possible combination of pairs of the seven publishers' tests originally included in the ATS. For purposes of this study only the fourth- and fifth-grade item response data for four reading comprehension subtests and three vocabulary subtests were selected.

The four publisher's tests included the California Achievement Tests (CAT; CTB/McGrawHill, 1970), Comprehensive Tests of Basic Skills (CTBS; CTB/McGraw-Hill, 1968), SRA 
Achievement Series (SRA; Science Research Associates, Inc., 1971), and Sequential Tests of Educational Progress (STEP; Educational Testing Service, 1969). The SRA vocabulary item response data were not available except for the 12 vocabulary-in-context items embedded within the reading comprehension subtest. These 12 items were included with the regular 48 reading comprehension items, creating a 60 -item SRA reading comprehension subtest, which was used in the subsequent analyses. The number of items and the appropriate grade levels by test, form, and level are given in Table 1.

Table 2 depicts the ATS data collection procedure for the three publishers' vocabulary subtests used in this study. In Table 2 each of the six cells represents an independent sample of examinees who were administered the pair of vocabulary subtests corresponding to the row and column headings. Cells above the diagonal refer to fifth-grade samples and cells below the diagonal refer to fourth-grade samples. For example, Cell a represents an independent sample of fifthgrade students who were administered the CAT and CTBS vocabulary subtests. Since each vocabulary subtest was paired with the other two vocabulary subtests at both grade levels, four in- dependent data sets (replications) were associated with each vocabulary subtest, e.g., two fifth-grade samples (Cells a and b) and two fourth-grade samples (Cells $d$ and e) were administered the CAT vocabulary subtest. A similar design was associated with the reading comprehension subtests except that a fourth publisher's test, SRA, was also considered. Consequently, there were six independent sets of examinee data, three at each grade, for each of the four publishers' reading comprehension subtests. Ignoring the order of administration, the sample sizes, ranging from 1,372 to 1,921 , for each pair of publishers' tests are given in Table 3.

\section{Model-Data Fit}

Preliminary data were obtained concerning the differing discrimination values of the items, and the speededness and the unidimensionality of each of the standardized achievement tests. The preliminary data provided information about the limits of applicability of the Rasch model, even though violations of the underlying Rasch assumptions could not be systematically varied. That is, information about the degree of

Table 1

Number of Items and Appropriate Grade Levels by Test, Level and Form

\begin{tabular}{lccccc}
\hline \hline & & & & \multicolumn{2}{c}{ Number of Items } \\
Test & Leve1 & Form & $\begin{array}{c}\text { Appropriate } \\
\text { Grades }\end{array}$ & Vocabulary & Reading \\
\hline CAT & 3 & A & 4,5 & 40 & 42 \\
CTBS & 2 & Q & 4,5 & 40 & 45 \\
STEP & 4 & A & $4,5,6$ & 30 & 30 \\
SRA & Blue & E & 4,5 & $42^{\mathrm{a}}$ & $60^{\mathrm{b}}$ \\
\hline
\end{tabular}

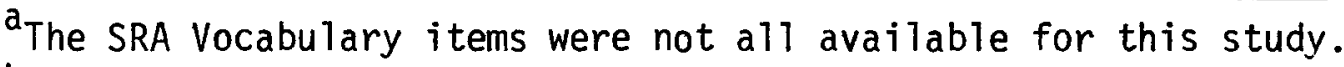
$b_{\text {Includes the }} 48$ regular reading comprehension items plus the 12 vocabulary-in-context items embedded within the reading comprehension subtest. 
Table 2

$\begin{aligned} & \text { Representation of the ATS Data Collection Samples } \\
& \text { for the Three Vocabulary }\end{aligned}$
\begin{tabular}{lccc} 
& CATts Used in this Study \\
\hline CAT & CTBS & STEP \\
CTBS & d & a & b \\
STEP & e & $f$ & $c$
\end{tabular}

Note. Fifth grade samples are above the
diagonal and fourth grade samples are
below the diagonal.

misfit, depending upon the results, gives some idea about the limits to which the Rasch assumptions can be violated in achieving objective measurement.

Unidimensionality. By inspecting the eigenvalues from the factor analysis of interim tetrachoric correlations computed on the combined fourth-and fifth-grade data, a check on the reasonableness of the unidimensionality of each subtest was made. The tetrachoric correlations require normality assumptions, which are not necessary to the Rasch model. Also, with multiple-choice items the normality assumption cannot be expected to hold because of the effects of guessing. Nonetheless, the factoring of the tetrachoric correlation matrices provides some indication of the plausibility of the unidimensionality assumption of the Rasch model. Certainly, if several large factors were indicated in the factor analysis, the assumption of unidimensionality would be highly suspect.

The particular factor extraction technique employed was the principal axis method with estimated communalities in the diagonal. The communality of each item was estimated by the squared multiple correlation with every other item. In addition to the factor analysis of each subtest, correlations between equated tests are also provided.

Nonspeededness. A rough notion as to the speededness of each subtest is given by the proportion of combined fourth- and fifth-grade ex-

Table 3

Sample Sizes for the Various Test Pairs

\begin{tabular}{lcccc}
\hline & CAT & CTBS & STEP & SRA \\
\hline CAT & & 1,921 & 1,397 & 1,492 \\
CTBS & 1,896 & & 1,784 & 1,638 \\
STEP & 1,447 & 1,743 & & 1,607 \\
SRA & 1,372 & 1,662 & 1,552 & \\
\hline Note. & $\begin{array}{l}\text { Grade 5 sample sizes are above the diagonal } \\
\text { and Grade 4 sample sizes are below the } \\
\text { diagonal. }\end{array}$ & & &
\end{tabular}


aminees failing to respond to each item. Of course, some speededness is expected for most standardized achievement tests. Typically, a nonspeeded test is considered to be one in which the proportion omitting the last few items is about .10 or less. In the long run, however, the differential amount of speededness between tests to be equated is likely to be a more important factor than the absolute degree of speededness, because an examinee's test behavior is appreciably different for two differentially speeded tests than for two equally speeded tests.

Equal discriminations. As previously mentioned, four independent sets of examinees' item response data (two at each grade) were available for each of the three publishers' vocabulary subtests. For each set of examinee data the proportion of very good-fitting items, those with discrimination values in the range .80 to 1.20 (Panchapakesan, 1969; Rentz \& Bashaw, 1975), was calculated. The average of the four proportions for each of the publishers' tests provided an overall picture of the extent to which the assumption of equal discrimination across all items was met. Proportions of items with discrimination values less than $.50, .50$ to $.79, .80$ to $1.20,1.21$ to 1.50 , and greater than 1.50 were also calculated for the equating samples. A similar procedure was followed for each of the four publishers' reading comprehension subtests except that six independent sets of examinees' item response data were available.

\section{Person-Free Item Calibration}

Evaluating the person-free item calibration property of the Rasch model involved determining how stable the item parameter estimates were when estimated across different groups of examinees. All Rasch item calibrations were performed using the BICAL computer program (Wright \& Mead, 1977). Six independent calibrations of the Rasch item parameters (three at each grade) were obtained for the reading comprehension subtests of the four publishers' test batteries. Only four independent calibrations were possible for the vocabulary subtests. In order to save on computer costs, the items for each test pair were commonly calibrated (recall that each examinee was administered two test batteries), i.e., the two tests were treated as one long test. This was done separately for the reading and vocabulary subtests of each battery. For both subtests of each publisher's test the intercorrelations, standard deviations, and means of the independent item parameter estimates were computed.

\section{Item-Free Person Measurement}

To assess the item-free person measurement property of the Rasch model, a paradigm depicting an equating situation that uses an anchor test must first be described. Generally, the paradigm involved treating the ATS data as though the easiest test, along with an anchor test, was administered to only fourth-grade students and as though the hardest test, along with the same anchor test, was administered only to fifth-grade students. Specifically, for the three vocabulary subtests the CTBS was selected as the anchor test; the CAT, using fifth-grade data, was equated to the STEP; while STEP used only fourth-grade data. For the four reading comprehension subtests two equating paradigms were simulated, both involving the CAT as the anchor test. Both the SRA and STEP using Grade 5 data were equated to the CTBS, while the CTBS used only Grade 4 data. A schematic representation of equating the easier STEP vocabulary subtest to the more difficult CAT vocabulary subtest is shown in Figure 1.

In general, the difference between the difficulty levels ( $p$-values) for the easiest and hardest tests was about .06 at each grade level. Also, in terms of $p$-values, the average difference between fourth and fifth graders was about .09 . Thus, the difference between the groups and the tests were not extreme, as in the Slinde and Linn studies, yet they were not all that small.

After calibrating the item parameters for pairs of tests to be equated, the Rasch log ability esti- 


\section{Figure 1}

Schematic Representation of Equating the Two Vocabulary Subtests

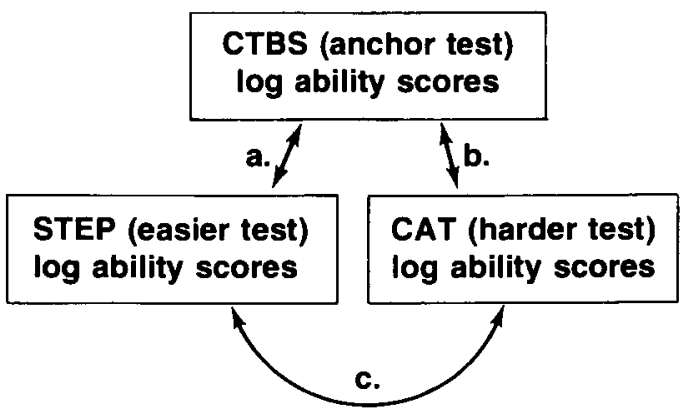

a. Equated thru their common item calibration using grade 4 examinees.

b. Equated thru their common item calibration using grade 5 examinees.

c. Equated using the 2 sets of anchor test (CTBS) log difficulty estimates.

mates for the two tests were then placed on the same scale by using the two sets of difficulty parameter estimates for the anchor test. Since, as $N$ approaches infinity, the scales for the two sets of difficulty estimates have the same variance and the correlation between the estimates is 1.0 , there is only a difference in the origin of the two scales. Thus, the difference between average difficulties estimates the additive constant, placing not only the difficulty estimates but also the ability estimates of the two tests to be equated on the same scale. For example, to equate the two vocabulary subtests, the mean of the CTBS difficulty estimates based on the CAT sample was subtracted from the mean of the CTBS difficulty estimates based on the STEP sample. The difference was then added to all CAT difficulty estimates to place CAT item difficulties on the same scale as STEP. A similar procedure was followed for the two pairs of reading comprehension subtests.
Evaluating the item-free person measurement property of the Rasch model involved noting that the equated easy and difficult tests actually had both been administered to a group of fourth-grade students and a group of fifth-grade students. Therefore, for these two groups of students, summary statistics for the Rasch log ability estimates were computed for each of the equated easy and difficult tests. In particular, the means, standard deviations, and correlations of the log ability estimates for the two equated tests provided data pertinent to item-free person measurement.

\section{Results and Discussion}

\section{Model-Data Fit}

Unidimensionality. The first 10 eigenvalues of the matrices of tetrachoric correlations among the items of each subtest are reported in Table 4. With one exception the percentage of common variance accounted for by the first factor for each of the subtests ranged from $65 \%$ to $75 \%$. The exception involved the SRA reading comprehension subtest, which had only $55 \%$ of the variance accounted for by the first factor. Since the second and successive latent roots were of similar magnitude and substantially smaller than the first, one very large dominant factor was associated with each subtest. Conventional rules for determining the number of factors indicated that there was at least one minor factor associated with all but one of the subtests, however.

Nonspeededness. The proportion of examinees failing to respond to each item was used to provide an indication of the speededness of the subtests. The proportion of omits for each of the subtests was, with rare exception, nondecreasing with respect to item number, i.e., if the proportion of omits was .06 for Item 7 , then the proportion of omits was at least .06 for Item 8 . Reported in Table 5 are the distributions of the proportion of omits for each subtest. An examination of the data reported in Table 5 indicates that the four reading subtests and 


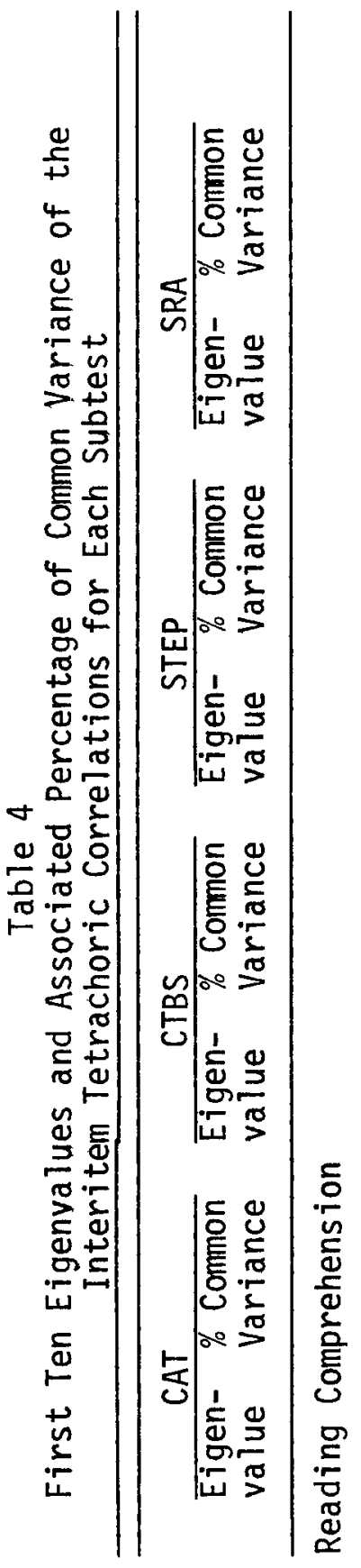

"mळ@ ம்

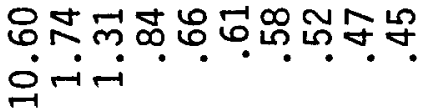

ஊNํำ

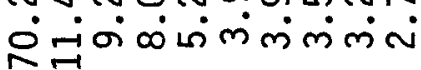

๓⿴囗十 . ! !? ! ! نं ๑

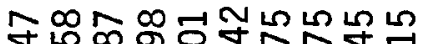
\%?

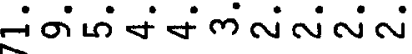

유요요 !.?!? ? ? oi

$m L \forall \sqcap \omega \sqcap L \infty N m$

जकमल

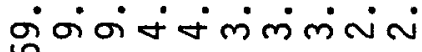
on

$00 \rightarrow N \omega \rightarrow N+9 \infty \frac{\pi}{8}$ थिण .?. ! ? ! ? $m \rightarrow-$
뚜 i.

Oᄋᄋm ?!? ? ? ?

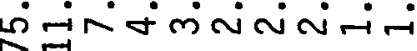
क幺

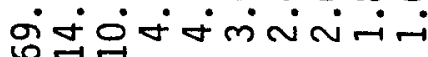

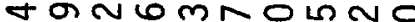
trNLnm $\mathrm{m} N \mathrm{~N}$ $\infty-\dot{1}$ 
Table 5

Distributions of the Proportion of Omits in Terms of the Proportion of Items in Each of the Subtests

\begin{tabular}{|c|c|c|c|c|c|c|c|}
\hline \multirow{2}{*}{$\begin{array}{c}\text { Proportion } \\
\text { of Omits }\end{array}$} & \multicolumn{3}{|c|}{ Vocabulary } & \multicolumn{4}{|c|}{ Reading } \\
\hline & $\overline{\mathrm{CAT}}$ & CTBS & STEP & $\overline{\mathrm{CAT}}$ & CTBS & SRA & STEP \\
\hline $\begin{array}{l}.00-.05 \\
.06-.10 \\
.11-.15 \\
.16-.20 \\
.21-.25 \\
.26-.30\end{array}$ & $\begin{array}{l}.600 \\
.075 \\
.100 \\
.075 \\
.050 \\
.100\end{array}$ & $\begin{array}{l}.775 \\
.225\end{array}$ & $\begin{array}{l}.667 \\
.133 \\
.100 \\
.100\end{array}$ & $\begin{array}{l}.810 \\
.190\end{array}$ & $\begin{array}{l}.889 \\
.111\end{array}$ & $\begin{array}{l}.667 \\
.083 \\
.100 \\
.117 \\
.033\end{array}$ & $\begin{array}{l}.633 \\
.167 \\
.133 \\
.067\end{array}$ \\
\hline
\end{tabular}

the three vocabulary subtests were differentially speeded with respect to their content areas. In fact, the CAT vocabulary subtest was extremely speeded. The proportion of omits for the last six items of this test ranged from .21 to .30 . Thus, not only was the assumption of nonspeededness not met to a reasonable approximation with most of the subtests but the degree of speededness was considerably different between the subtests of a given content area.

Equal discriminations. As described earlier, four independent sets of examinee item data were available for each of the vocabulary subtests, and six sets of data were available for each of the reading comprehension subtests. For each subtest half of the independent sets of data involved Grade 5 examinees, while the other half involved Grade 4 examinees. Across the replications the average proportion of items having discrimination values in the range .80 to 1.20 were $.575, .506$, and .633 for the CAT, CTBS, and STEP vocabulary subtests, respectively. For the CAT, CTBS, STEP, and SRA reading comprehension subtests, the average proportions across the six replications were $.409, .444, .550$, and .367 , respectively. On the average, the vocabulary subtests had a larger proportion of very good fitting items than the reading comprehension subtests, but for none of the subtests was the model-data fit exceptionally good in terms of the discrimination index. Distributions of the item discrimination values, in terms of the proportion of items in each subtest, are reported in Table 6. These distributions were constructed using only those samples that were involved in the equating paradigms. As noted from the table, several subtests contained a few items for which the discrimination value deviated by .51 or more from the standard value of 1.00 .

Implications. Results pertaining to modeldata fit indicated that the assumption of unidimensionality for each of the reading subtests was not met, strictly speaking, al though one very large major factor was associated with each of the subtests. In addition, the assumptions of nonspeededness and uniform item discriminations were violated to a substantial degree. With only four options per multiple-choice question it is probable that guessing was also a factor. This is likely to be true for many national standardized achievement tests, since such tests are developed along the same general guidelines and the examinees being tested span a wide range of ability. Consequently, the Rasch model is apt to be considered suspect when the sole criterion involves satisfying the assumptions of the model.

Although in terms of satisfying the antecedent conditions of the model, fit is an important consideration, the issue of primary importance is whether the model achieves what it purports to, even though the underlying assumptions of the model are not met. In fact, the empirical portion 
Table 6

Distributions of the Item Discrimination Values

in Terms of the Proportion of Items in Each of the Subtests

\begin{tabular}{llllll}
\hline Test $^{\mathrm{a}}$ & \multicolumn{5}{c}{ Discrimination Values } \\
\cline { 2 - 6 } & .49 or less & $.50-.79$ & $.80-1.20$ & $1.21-1.50 \geqslant 1.51$ \\
\hline Vocabulary Subtests & \multicolumn{5}{c}{ or } \\
CAT (CTBS-5) & & .150 & .600 & .250 & \\
STEP (CTBS-4) & .033 & .200 & .500 & .267 & \\
CTBS (CAT-5) & .125 & .100 & .575 & .200 & \\
CTBS (STEP-4) & .100 & .150 & .425 & .300 & .025 \\
Reading Subtests & & & & & \\
CTBS (CAT-4) & .089 & .089 & .444 & .244 & .133 \\
STEP (CAT-5) & .067 & .133 & .467 & .333 & \\
SRA (CAT-5) & .050 & .217 & .333 & .367 & .033 \\
CAT (CTBS-4) & .167 & .143 & .381 & .286 & .024 \\
CAT (STEP-5) & .095 & .095 & .381 & .429 & \\
CAT (SRA-5) & .071 & .167 & .571 & .190 & \\
\hline
\end{tabular}

\section{${ }^{a}$ Enclosed in parentheses are the calibration sample.}

of the present study was largely concerned with just this issue: Is the Rasch model robust with respect to existing standardized reading achievement tests? The consequences or desirable outcomes of the Rasch model were defined in terms of person-free item calibration and item-free person measurement, and the degree of misfit was that which is naturally associated with the standardized reading achievement tests used in this study.

\section{Person-Free Item Calibration}

The intercorrelations, means, and standard deviations of Rasch difficulty estimates were computed for each subtest across the several replications. Since two grade levels were involved, both random (within grade) and nonrandom (between grade) replications were considered. For the vocabulary subtests the summary measures are reported in Table 7 and for the reading comprehension subtests the indices are reported in Table 8.
Correlation of item difficulties. As can be seen in both tables, the intercorrelations were extremely high, with only a few coefficients less than .98 and none less than .94 , with the STEP reading comprehension subtest providing the lowest intercorrelations (five of the six correlations were less than .97). Thus, the ordering of Rasch difficulty estimates for each subtest was reasonably constant across the several replications.

Standard deviation of difficulties. For each subtest the standard deviations of the difficulty estimates were very similar. Typically, the range of these standard deviations across the replications was about .08. With one notable exception, there was no tendency for the standard deviations to be higher or lower at one ability level (grade) than another. The exception involved the CAT reading comprehension subtest. On the average, the CAT standard deviations based on Grade 4 data were about .13 log unit smaller than those based on Grade 5 data. Despite the one exception, the unit of the scale of measure- 
Table 7

Intercorrelations, Means and Standard Deviations of the Vocabulary Subtest Item Difficulty Estimates

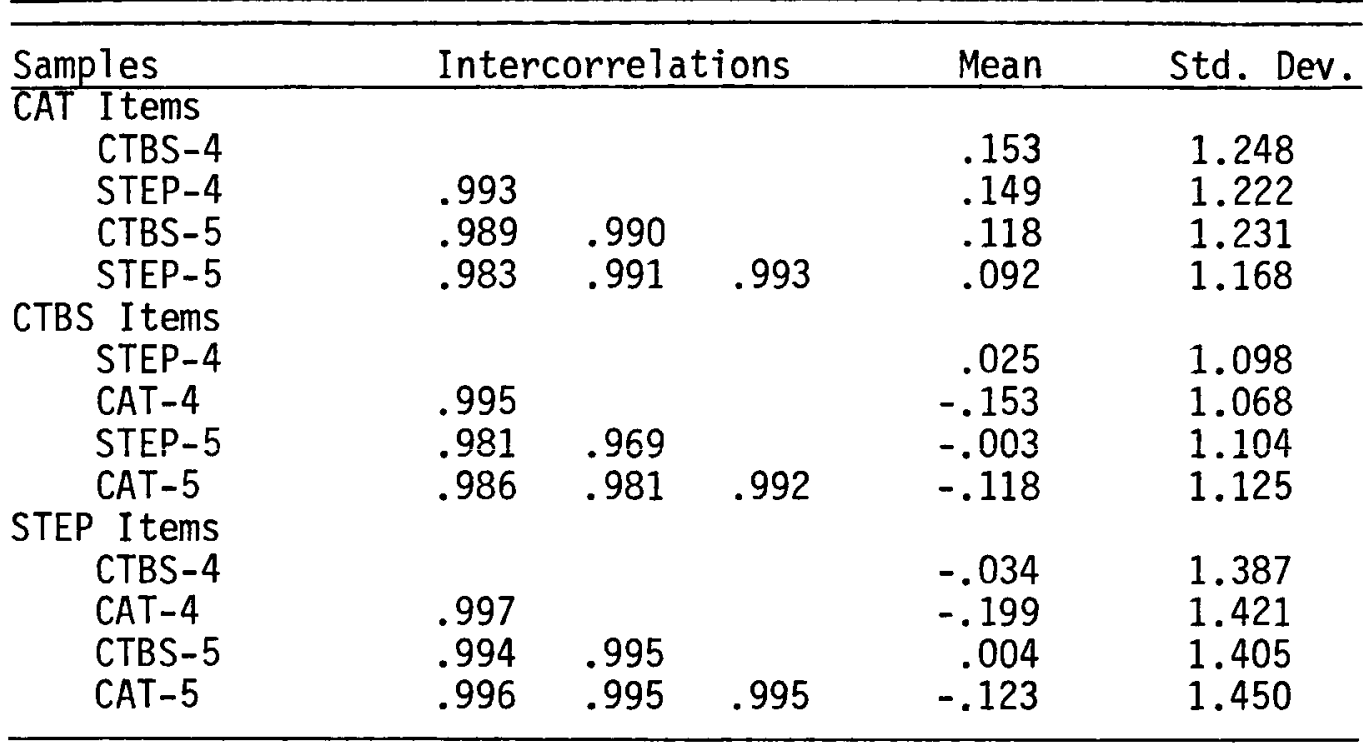

ment for item difficulty was essentially the same across the random and nonrandom replications.

Mean item difficulties. Because each subtest was commonly calibrated with another subtest, it is inappropriate to compare the means of the difficulty estimates for equality across all replications of a given test. The reason for this is that in order to overcome the indeterminacy in the scale of difficulty, the mean of all commonly calibrated items (e.g., CAT and CTBS items) was arbitrarily set equal to zero. Consequently, the mean for any subset of commonly calibrated items (e.g., just the CAT items) is dependent on what other items are included in the calibration. Since the same set of items were commonly calibrated for certain pairs of nonrandom replications (different grade levels), comparisons of these means was appropriate.

For example, the means of the CAT difficulty estimates, which were based on the CTBS-4 and CTBS-5 samples, can be compared; and similarly, for the STEP-4 and STEP-5 samples. Therefore, two comparisons of means were made for each of the three vocabulary subtests and three comparisons were made for each of the four reading comprehension subtests. Most of the differences between the 18 pairs of means were about $.04 \log$ unit or less, but the STEP reading comprehension subtest provided the largest mean differences of .09 and .08 . Considering the size of the standard deviations, the origin of the scale of measurement of the difficulty estimates was very similar across the nonrandom replications.

Implications. To a reasonable approximation, the results concerning person-free item calibration indicated that across the random and nonrandom replications, the ordering of Rasch difficulty estimates was constant and the means and the standard deviations of the difficulty estimates were very similar.

The results indicating that the Rasch difficulty estimates were reasonably stable would seem especially convincing, since no attempt was made to delete poor-fitting items, whereas the data concerning the assumptions of the model indicated considerably less than satisfactory modeldata fit. But it must also be emphasized that 
Table 8

Intercorrelations, Means, and Standard Deviations of the Reading Comprehension Subtest Item Difficulty Estimates

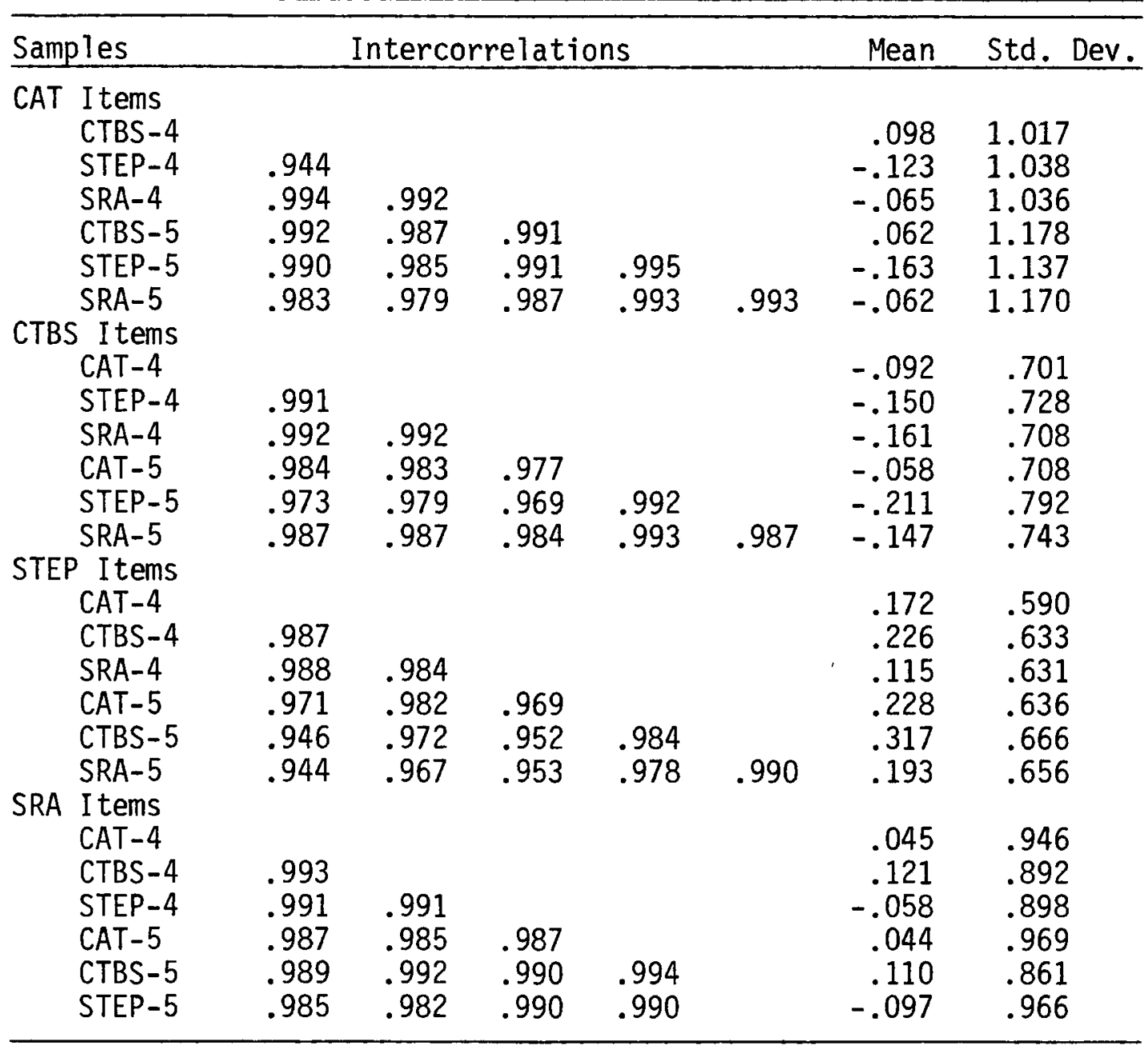

very large samples $(1,372$ to 1,921$)$ were used in this study; and as suggested by the Rentz and Bashaw (1975) and Tinsely and Dawis (1975) studies, the effect of using very large samples may have been the reduction of any distortion in the person-free item calibration results, which would otherwise have occurred if small samples sizes (100 to 500$)$ had been used. All in all, the results of this study and of previous studies (Anderson, Kearney, \& Everett, 1968; Rentz \&
Bashaw, 1975; Tinsley \& Dawis, 1975) strongly suggest that Rasch difficulty estimates are relatively independent of the ability level of the calibration sample for groups not widely different in ability. The range of ability of the calibration sample in relation to the difficulty level of the items, the degree of model-data fit, and the size of the calibration sample undoubtedly interact to determine the extent to which invariance is achieved, however. 


\section{Item-Free Person Measurement}

Within the two content areas (reading comprehension and vocabulary), three equating paradigms using an anchor test were described. Since each pair of equated tests was actually administered to a sample of Grade 4 and a sample of Grade 5 students, the means, standard deviations, and correlations of the Rasch log ability estimates for each of the three equated test pairs provided data relevant to item-free person measurement. The summary measures for the vocabulary subtests are reported in Table 9 , and for the reading comprehension subtests the measures are reported in Tables 10 and 11.

Correlation of ability estimates. For all three equated test pairs, and for both grade levels, the correlations between Rasch log ability estimates were relatively high (ranging from .78 to .85 ), indicating that the examinees were ordered similarly on the equated test pairs. Although consistently lower, the correlations between Rasch log ability scores differed by less than .02 from the corresponding raw score correlations. Thus, little, if any, information was lost due to use of the Rasch model to equate the various test pairs.

Mean and standard deviation of ability estimates. The remaining results pertaining to item-free person measurement were generally favorable, but not ideal. Perhaps the only equated test pair which can be treated as statistically equivalent throughout the range of measured ability (Grades 4 and 5) was the CTBS and SRA reading comprehension subtests. Even for this test pair the difference between the Grade 5 $\log$ ability means was -.076 , which was more than four standard errors from zero. This is likely to be more a purely theoretical rather than a practical difference, however, since the mean difference was rather small in relation to the size of the standard deviation of log ability scores for each test.

For the two equated vocabulary subtests the difference between the two means and the two standard deviations was negligible for the Grade 4 data; but for the Grade 5 data the difference between means was .13 and the difference between standard deviations was .10. Although the Grade 5 differences are not exceptionally large, they seem to indicate that there was less than an ideal equating of the two vocabulary subtests. The equating results for the CTBS and STEP reading comprehension subtests clearly indicated that these two tests did not provide statistically equivalent $\log$ ability estimates throughout the range of measured ability. The

Table 9

Comparison of the Log Ability Scores for the Equated CAT and STEP Vocabulary Subtests

\begin{tabular}{|c|c|c|c|c|}
\hline $\begin{array}{l}\text { Grade and } \\
\text { Statistic }\end{array}$ & CAT & STEP & $\begin{array}{c}\text { Difference } \\
\text { Score }\end{array}$ & Correlation $^{\mathrm{a}}$ \\
\hline \multicolumn{5}{|l|}{ Grade 4 Data } \\
\hline $\begin{array}{l}\text { Mean } \\
\text { Std. Error } \\
\text { Std. Dev. } \\
\text { Grade } 5 \text { Data }\end{array}$ & $\begin{array}{l}0.339 \\
0.036 \\
1.361\end{array}$ & $\begin{array}{l}0.336 \\
0.036 \\
1.358\end{array}$ & $\begin{array}{r}-0.027 \\
0.022 \\
0.823\end{array}$ & $\begin{array}{l}.817 \\
(.825)\end{array}$ \\
\hline $\begin{array}{l}\text { Mean } \\
\text { Std. Error } \\
\text { Std. Dev. }\end{array}$ & $\begin{array}{l}1.091 \\
0.040 \\
1.479\end{array}$ & $\begin{array}{l}0.960 \\
0.037 \\
1.380\end{array}$ & $\begin{array}{l}0.131 \\
0.023 \\
0.834\end{array}$ & $\begin{array}{l}.832 \\
(.848)\end{array}$ \\
\hline
\end{tabular}

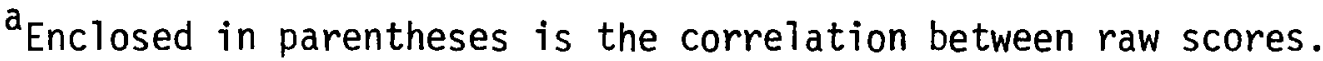


Table 10

Comparison of the Log Ability Scores for the Equated CTBS and SRA Reading Subtests

\begin{tabular}{lrrrc}
\hline $\begin{array}{l}\text { Grade and } \\
\text { Statistic }\end{array}$ & CTBS & SRA & $\begin{array}{c}\text { Difference } \\
\text { Score }\end{array}$ & Correlation $^{a}$ \\
\hline Grade 4 Data & & & & \\
$\quad$ Mean & -0.299 & -0.285 & -0.014 & .827 \\
$\quad$ Std. Error & 0.027 & 0.028 & 0.016 & $(.839)$ \\
$\quad$ Std. Dev. & 1.116 & 1.136 & 0.662 & \\
Grade 5 Data & & & & \\
$\quad$ Mean & 0.139 & 0.216 & -0.076 & .855 \\
Std. Error & 0.029 & 0.030 & 0.016 & $(.858)$ \\
Std. Dev. & 1.184 & 1.229 & 0.651 & \\
\hline
\end{tabular}

${ }^{2}$ Enclosed in parentheses is the correlation between raw scores.

Grade 5 mean difference was -.08 , which was not especially large; but the Grade 4 mean difference was -.21 , and the difference between the standard deviations was almost .10. The difference of -.21 was approximately one-fifth of the standard deviation of the log ability scores for each test, and therefore item-free person measurement was not achieved with respect to Grade 4 data.

The person-free item calibration results associated with STEP could account for the poor item-free person-measurement results for the STEP and CTBS reading comprehension subtests. STEP provided the lowest correlations and the largest mean differences between the difficulty estimates computed for each subtest. In addition, the correlational data and the information from content specialists obtained in the original ATS (Bianchini \& Loret, 1974) indicated that the STEP reading subtest may be measuring something apart from what the remaining reading comprehension subtests are measuring.

Other criteria. The item-free person measurement results can also be evaluated in relation to two other criteria - the standard error of measurement and the log ability difference between adjacent raw scores. For example, the dif- ference between adjacent raw scores in the middle of the distribution was about $.14 \log$ unit for the CAT vocabulary subtest and about .20 $\log$ unit for the STEP vocabulary subtest. As expected, the $\log$ ability differences were considerably larger at the extremes of the score distribution. Therefore, the Grade 5 mean difference of $.13 \log$ units for the two equated vocabulary subtests represented a difference that was typically less than one raw score point in the middle of the distribution and considerably less than one raw score point at the extremes.

Furthermore, the standard error of measurement for scores in the middle of the distribution was about .38 for the CAT and about .45 for STEP. Thus, the mean difference of .13 was no larger than a third of the error of measurement for either subtest. Although this is not trivial, it is probable that for many testing situations mean differences as large as the one for the two equated vocabulary subtests could reasonably be tolerated in relation to the types of decisions likely to be made with equated tests.

With the exception of the Grade 4 mean differences for the CTBS and STEP reading comprehension subtests, the remaining mean differences were rather small (if not trivial) in terms of their absolute value, the standard deviation of 
Table 11

Comparison of the Log Ability Scores for the Equated CTBS and STEP Reading Subtests

\begin{tabular}{lrrrc}
\hline $\begin{array}{l}\text { Grade and } \\
\text { Statistic }\end{array}$ & CTBS & STEr & $\begin{array}{c}\text { Difference } \\
\text { Score }\end{array}$ & Correlation $^{2}$ \\
\hline Grade 4 Data & & & & \\
$\quad$ Mean & -0.065 & 0.146 & -0.211 & .782 \\
$\quad$ Std. Error & 0.027 & 0.024 & 0.017 & $(.789)$ \\
Std. Dev. & 1.106 & 1.012 & 0.706 & \\
Grade 5 Data & & & & .799 \\
Mean & 0.470 & 0.554 & -0.084 & $(.801)$ \\
Std. Error & 0.028 & 0.027 & 0.017 & \\
Std. Dev. & 1.173 & 1.124 & 0.730 & \\
\hline
\end{tabular}

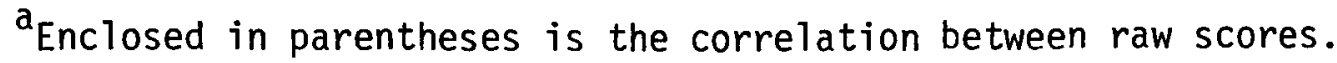

the $\log$ ability scores, the $\log$ ability difference between adjacent raw scores, and the standard error of measurement. The Grade 4 mean difference of -.21 for the CTBS and STEP reading subtests, however, was approximately onefifth of a standard deviation of the log ability scores for either test, representing a difference in the middle of the distribution that was as much as two score points between adjacent raw scores and as large as two-thirds of the error of measurement. Thus, the equating of the CTBS and STEP reading comprehension subtests was less than satisfactory for Grade 4 examinees.

Other than this exception, however, the Rasch model using the anchor test procedure provided person measurement with existing standardized reading achievement tests, which (to a rough approximation) was item free throughout the range of measured ability. These results are encouraging, since there was considerably less than satisfactory model-data fit in terms of the assumptions of uniform item discriminations; nonspeededness; unidimensionality; and, very possibly, zero asymptotes (no guessing).

\section{Conclusions}

The information pertaining to model-data fit indicated that the assumption of unidimen- sionality for each of the reading subtests was not met, strictly speaking, although one very large major factor was associated with each subtest. In addition, the assumptions of nonspeededness and uniform item discriminations were violated to a substantial degree. It is likely that with only four options per multiple-choice question, guessing was also a factor.

Despite the considerable lack of model-data fit, the Rasch difficulty estimates for the vocabulary and reading comprehension subtests were reasonably invariant for replications with random samples and samples differing in ability by one grade level. Furthermore, with the exception of the data for one test pair and one grade level, the Rasch model using the anchor test procedure provided a reasonably satisfactory means of equating three test pairs on the log ability scale for examinees at two grade levels. Consequently, the Rasch model provided approximately item-free person measurement in all but one case with existing standardized reading. achievement tests, even though the assumptions of the model were not adequately met.

The item-free person results of this study were not as good as those for the Wright (1968), Whitely and Dawis (1974), and Tinsley and Dawis (1977) studies, which were judged to pro- 
vide strong support for the claim for item-free person measurement with the Rasch model, but they do lend reasonable support for the claim. It may be that the almost ideal item-free person results reported in those studies were the result of not using cross-validations with samples that differed in ability, whereas this study and the Slinde and Linn $(1978,1979)$ studies did involve cross-validations with differing ability level samples.

The results of this study suggest that the Rasch model has greater utility for the purpose of vertical equating than was apparent in the previous Slinde and Linn studies. The results reported here, however, are not inconsistent with the results reported in the earlier studies by Slinde and Linn, since their tests of the Rasch model were quite severe. The comparisons between the nonparallel tests in the Slinde and Linn studies were more extreme in terms of the wide separation of the high- and low-ability groups than are apt to be encountered when equating tests over different grades. They also indicated that better results might have been expected with an anchor test procedure. In this study an anchor test procedure was used and the difference between the high- and low-ability level calibration samples was one grade level. In terms of the standard deviation unit on the $\log$ ability scale, the separation between high- and low-ability groups for the hard and easy tests was about five to six times larger for the Slinde and Linn studies than that used here.

Apart from considerations of model-data fit, it may be that item-free person measurement can be achieved with the Rasch model to the extent that samples of examinees differ by the equivalent of one or two grade levels. This does not preclude the possibility of equating several levels of a standardized achievement test (assuming unidimensionality), since the equating can still be accomplished grade by grade with the anchor test procedure, although the extent of "linking errors" is not known and hence should be investigated.
The results of this study indicate that there is reason to be optimistic about using the Rasch model to scale existing test data in order that objective measurement can be achieved. The twoand three- parameter logistic latent trait models may be expected to provide even better results, however, since these models attempt to take into account more information about each item by incorporating additional item parameters for discrimination and for the lower asymptote. In addition, the results of the Slinde and Linn studies $(1978,1979)$ and those for monte carlo studies (Dinero \& Haertel, 1977; Hambleton \& Traub, 1971) suggest that the Rasch model may not be adequate for low-ability examinees; hence, models containing additional item parameters may be required in such circumstances. The higher order models, however, require quite large sample sizes with presently used estimation techniques, and consequently they may have limited application (e.g., regional or national testing). In addition, the conditional maximum likelihood estimation procedure cannot be strictly applied to test data when the discrimination values are not equal, since the sufficient statistic will depend on unknown and unobservable parameters (Hambleton \& Cook, 1977). Thus, it is not known whether there is any gain in using the higher order models over the Rasch model.

A modified version of the Rasch model (Waller, 1976) may prove to be very useful in those situations in which the Rasch model is not, since the procedure attempts to remove the effects of random guessing from the estimates of difficulty and ability without altering the basic properties of the Rasch model (e.g., mathematical and computational simplicity and uniqueness). The modified procedure is an application of the abilities removing random guessing (ARRG) model developed for the twoparameter latent trait model (Waller, 1974). The assumption underlying the ARRG procedure is that examinees who guess in an essentially random manner do so on those items that are too 
difficult for them. Thus, the ARRG procedure removes from the estimation procedure those item-person interactions in which a person responds to an item estimated to be very difficult for that person. Consequently, the modified Rasch model can be considered a rough approximation to tailored testing, and research using this model should hence prove useful.

\section{References}

Anderson, J., Kearney, G. E., \& Everett, A. V. An evaluation of Rasch's structural model for test items. British Journal of Mathematical and Statistical Psychology, 1968, 21, 231-238.

Bianchini, J. C., \& Loret, P. G. Anchor test study: Final report (Project Report and Vols. 1-30) and Anchor test study: Supplement (Vols. 31-33), 1974. (ERIC Document Reproduction Service Nos. ED 092 601-ED 092 634)

CTB/McGraw-Hill. Comprehensive Tests of Basic Skills (1968 ed.). Monterey, CA: Author, 1968.

CTB/McGraw-Hill. California Achievement Tests (1970 ed.). Monterey, CA: Author, 1970.

Dinero, T. E., \& Haertel, E. Applicability of the Rasch model with varying item discriminations. Applied Psychological Measurement, 1977, 1, 581-592.

Educational Testing Service. Sequential Tests of Educational Progress (1969 ed.). Princeton, NJ: Author, 1969.

Hambleton, R. K., \& Cook, L. Latent trait models and their use in the analysis of educational test data. Journal of Educational Measurement, 1977, 14. 75-96.

Hambleton, R. K., \& Traub, R. E. Information curves and efficiency of three logistic test models. British Journal of Mathematical and Statistical Psychology, 1971, 24, 273-281.

Panchapakesan, N. The simple logistic model and mental measurement. Doctoral dissertation, University of Chicago, 1969.

Rasch, G. Probabilistic models for some intelligence and attainment tests. Copenhagen: Danmarks Institut Paedagogiske, 1960.

Rentz, R. R., \& Bashaw, W. L. Equating reading tests with the Rasch model (Vols. 1 \& 2). Athens: University of Georgia, Educational Research
Laboratory, August 1975. (ERIC Document Reproduction Service Nos. ED 127 330-ED 127 331)

Slinde, J. A., \& Linn, R. L. An exploration of the adequacy of the Rasch model for the problem of vertical equating. Journal of Educational Measurement, 1978, 15, 23-35.

Slinde, J. A., \& Linn, R. L. A note on vertical equating via the Rasch model for groups of quite different ability and tests of quite different difficulty. Journal of Educational Measurement, 1979, 16. 159-165.

Science Research Associates. SRA Achievement Series (1971 ed.). Chicago: Author, 1971.

Tinsley, H. E., \& Dawis, R. V. An investigation of the Rasch simple logistic model: Sample-free item and test calibration. Educational and Psychological Measurement, 1975, 35, 325-339.

Tinsley, H. E., \& Dawis, R. V. Test-free person measurement with the Rasch simple logistic model. Applied Psychological Measurement, 1977, 1, 483-487.

Waller, M. I. Removing the effects of random guessing from latent trait ability estimates (ETS RB 74-32). Princeton, NJ: Educational Testing Service, 1974.

Waller, M. E. Estimating parameters in the Rasch model: Removing the effects of random guessing (ETS RB 76-8). Princeton, NJ: Educational Testing Service, 1976.

Whitely, S. E., \& Dawis, R. V. The nature of objectivity with the Rasch model. Journal of Educational Measurement, 1974, 11, 163-178.

Wright, B. D. Sample-free test calibration and person measurement. Proceedings of the 1967 Invitational Conference on Testing Problems. Princeton, NJ: Educational Testing Service, 1968.

Wright, B. D., \& Mead, R. J. BICAL: Calibrating items and scales with the Rasch model (Research Memorandum No. 23). Chicago, IL: University of Chicago, Department of Education, Statistical Laboratory, 1977.

Wright, B.D., \& Panchapakesan, N. A procedure for sample-free item analysis. Educational and Psychological Measurement, 1969, 29, 23-48.

\section{Author's Address}

Send requests for reprints or further information to Jeffrey A. Slinde, Science Research Associates, Inc., 155 North Wacker Drive, Chicago, IL 60606. 